\title{
Óbito Decorrente da Síndrome de Hipersensibilidade ao Alopurinol (SHA) $)^{(*)}$
}

\section{Death Due to Allopurinol Hypersensivity Syndrome (AHS)}

\author{
Acir Rachid ${ }^{(1)}$, Francisco Luiz Gomide Mafra Magalhães ${ }^{(2)}$, Jean Rodrigo Tafarel ${ }^{(3)}$ e Rodrigo Schmitz ${ }^{(3)}$
}

\section{RESUMO}

A hiperuricemia pode causar desde a deposição de cristais de urato monossódico na cavidade articular até a "angústia clínica", provocando a prescrição desnecessária de fármacos. Neste caso, o paciente terá mais ganho de efeitos colaterais que benefícios. O alopurinol é o uricorredutor mais prescrito, sendo que reações de hipersensibilidade ocorrem em até $10 \%$ de seus usuários, com mortalidade de $20 \%$. Com este relato de caso objetivamos caracterizar a síndrome de hipersensibilidade ao alopurinol (SHA), enfatizando o correto uso dessa droga.

Palavras-chave: síndrome de hipersensibilidade ao alopurinol (SHA), hiperuricemia, efeitos colaterais.

\section{INTRODUÇÃO}

A elevação dos níveis séricos de ácido úrico pode causar desde a deposição de cristais de urato monossódico na cavidade articular até a "angústia clínica", que precipita o tratamento da hiperuricemia assintomática. Este tratamento envolve desde a utilização de uma dieta com baixas concentrações de purina até o uso de medicamentos uricosúricos ou uricorredutores ${ }^{(1)}$.

Medicações uricosúricas, como a probenecida, têm difícil adesão do paciente, pois são necessárias altas doses com várias tomadas diárias, além de não atuarem nos casos de insufi-

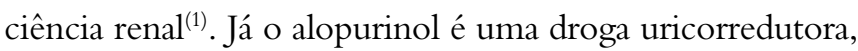
que permite ao doente uma única dose diária e, ao médico, a possibilidade de seu uso na insuficiência renal ${ }^{(2)}$.

No entanto, em razão provavelmente da má indicação no início do tratamento da hiperuricemia, notamos uma

\begin{abstract}
Hyperuricemia can lead to deposition of monosodium urate crystals in the joint cavity or to "physicians' anguish", causing prescription of unnecessary drugs. In this case, more side effects than benefits will be observed. Allopurinol is the most prescribed urate lowering drug and hypersensivity reactions occur in up to $10 \%$ of patients taking the drug, with mortality of $20 \%$. With this case report we intend to characterize the Allopurinol Hypersensivity Syndrome (AHS), emphasizing the right use of the drug.
\end{abstract}

Keywords: Allopurinol Hypersensivity Syndrome (AHS), hyperuricemia, side effect.

crescente lista de publicações revelando os efeitos tóxicos e fatais destes medicamentos. Até 10\% dos usuários de alopurinol apresentam reações auto-limitadas, como rash ou intolerância intestinal, mas reações severas também ocorrem e caracterizam a chamada síndrome de hipersensibilidade ao alopurinol (SHA), que provoca óbito em 20\% dos pacientes $^{(3,4)}$.

Desta forma, discutimos, a partir do relato de óbito de uma paciente com SHA, as características desta síndrome e as principais indicações para o tratamento da hiperuricemia.

\section{RELATO DE CASO}

Paciente feminina, de 65 anos de idade, procurou o serviço de clínica médica do Hospital de Clínicas de Curitiba, por apresentar dispnéia, tosse produtiva, febre e rash cutâneo disseminado e pruriginoso com cinco dias de evolução.

* Departamento de Clínica Médica da Universidade Federal do Paraná (UFPR). Recebido em 05/12/2003. Aprovado, após revisão, em 09/02/2004.

1. Professor emérito da UFPR. Professor sênior do departamento de clínica médica da UFPR. Livre-docente em Reumatologia e professor titular da disciplina de clínica médica da UFPR. Chefe do setor IV da enfermaria do departamento de clínica médica do Hospital de Clínicas da UFPR.

2. Médico especialista em clínica médica e medicina de urgência pela SBCM. Vice-chefe do setor IV da enfermaria do departamento de clínica médica do Hospital de Clínicas da UFPR. Mestrando em medicina interna da UFPR. Médico da unidade de urgência-emergência do Hospital de Clínicas da UFPR

3. Médico residente em clínica médica do Hospital de Clínicas da UFPR. 
A paciente era hipertensa, diabética, portadora de DPOC e há quatro semanas havia iniciado o uso de alopurinol $300 \mathrm{mg} /$ dia para quadro de artrite gotosa.

Ao exame físico apresentava-se em regular estado geral, confusa, dispnéica, hipocorada e cianótica. A pressão arterial era de $90 / 60 \mathrm{mmHg}$, pulso de $110 \mathrm{bpm}$, freqüência respiratória de $34 \mathrm{ipm}$ e temperatura axilar de $37,1^{\circ} \mathrm{C}$. As mucosas estavam discretamente secas, com jugulares e vasos sublinguais baixos, não havendo linfonodos palpáveis. A ausculta cardíaca possuía ritmo regular e bulhas hipofonéticas. A ausculta pulmonar revelava roncos e sibilos difusos. As extremidades de membros superiores estavam cianóticas e as falanges distais apresentavam tofos gotosos. Na pele havia rash máculo-papular com escoriações acometendo tronco e extremidades. Realizaram-se exames laboratoriais (Tabela 1) e ecografia de abdome total, que não evidenciou litíase renal ou nefropatia crônica.

Iniciou-se tratamento para broncopneumonia com ceftriaxona $2 \mathrm{~g}$ /dia, broncodilatarores inalatórios, corticóides e antihistamínicos, sendo também suspensas todas as medicações que a paciente usava previamente. A paciente necessitou de dopamina $(7 \mu \mathrm{g} / \mathrm{kg} / \mathrm{min})$ durante as primeiras 24 horas de internamento, permanecendo estável após sua retirada. O rash cutâneo apresentou melhora progressiva, evoluindo com descamação. Os níveis séricos de CK, CK-MB, transaminases e creatinina normalizaram.

No quinto dia de internamento a paciente evoluiu com celulite na região anterior do antebraço esquerdo, associada à leucopenia e neutropenia, sendo iniciados cefepima e oxacilina. Realizou-se um aspirado da medula óssea, o qual não obteve material, e biópsia, bem como tratamento com filgrastina (G-CSF - fator estimulador de colônia de granulócitos).

No sexto dia de internação, evoluiu com esforço respiratório e ausculta pulmonar com roncos e sibilos difusos, sendo necessário uso de ventilação mecânica. À noite sofreu parada cardiorrespiratória em assistolia, voltando a ritmo sinusal após cinco minutos de reanimação.

No sétimo dia, a paciente apresentou hipotensão não responsiva a volume, retornando-se ao uso de dopamina e posteriormente adrenalina. Nessa noite, sofreu três novas paradas cardiorrespiratórias em assistolia, evoluindo para óbito.

\section{DISCUSSÃO}

O alopurinol inicialmente foi sintetizado como droga quimioterápica, tendo pouco ou nenhum efeito sobre os tumores em que foi testado ${ }^{(5)}$. Nos últimos anos é o redutor
TABELA 1

EXAMES LABORATORIAIS NA ADMISSÃO DE PACIENTE

\begin{tabular}{|c|c|c|}
\hline \multicolumn{3}{|l|}{ Hemograma: } \\
\hline VG & $36,5 \%$ & \\
\hline $\mathrm{Hb}$ & $11,6 \mathrm{~g} / \mathrm{dl}$ & \\
\hline Leucócitos & $5,770 / \mathrm{mm}^{3}$ & \\
\hline Bastões & $25 \%$ & \\
\hline Eosinófilos & $0 \%$ & \\
\hline Plaquetas & $100,000 / \mathrm{mm}^{3}$ & \\
\hline Cálcio ionizado & $5,3 \mathrm{mg} / \mathrm{dl}$ & \\
\hline CK & $779 \mathrm{U} / \mathrm{L}$ & 6,395 (após 34 horas) \\
\hline CK-MB & $62 \mathrm{U} / \mathrm{L}$ & 284 (após 34 horas) \\
\hline TG0 (AST) & $191 \mathrm{U} / \mathrm{L}$ & \\
\hline TGP (ALT) & $95 \mathrm{U} / \mathrm{L}$ & \\
\hline Albumina & $3,4 \mathrm{~g} / \mathrm{dl}$ & \\
\hline RNI & 1,55 & \\
\hline Glicemia & $320 \mathrm{mg} / \mathrm{dl}$ & \\
\hline Creatinina & $3,3 \mathrm{mg} / \mathrm{dl}$ & \\
\hline Ácido úrico & $5,0 \mathrm{mg} / \mathrm{dl}$ & \\
\hline TSH & $0,252 \mu \mathrm{UI} / \mathrm{ml}$ & \\
\hline $\begin{array}{l}\text { Gasometria arteri } \\
\text { (com } 02 \text { a } 3 \mathrm{l} / \mathrm{min}) \\
\mathrm{pH}\end{array}$ & 7,34 & \\
\hline $\mathrm{Pa} 02$ & $94,6 \mathrm{mmHg}$ & \\
\hline $\mathrm{PaC02}$ & $17,7 \mathrm{mmHg}$ & \\
\hline Bicarbonato & $17,7 \mathrm{mmol} / \mathrm{L}$ & \\
\hline Sat02 & $97,7 \%$ & \\
\hline Parcial de urina: & & \\
\hline Proteínas & + & \\
\hline Hemoglobina & ++++ & \\
\hline Leucócitos & $11,000 / \mathrm{ml}$ & \\
\hline Hemácias & $100,000 / \mathrm{ml}$ & \\
\hline
\end{tabular}

de urato mais prescrito no tratamento da hiperuricemia e gota, embora seu uso em pacientes com hiperuricemia assintomática não seja justificável ${ }^{(6,7)}$. A SHA foi inicialmente descrita na década de 70, sendo a sua freqüência de $1 \mathrm{em}$ 260 pacientes, com tempo médio de uso do fármaco de 2 a 6 semanas $^{(3,4,6,8)}$.

Em 1986, Singer e Wallace definiram os critérios diagnósticos da SHA ${ }^{(4,6,8)}$ :

1. critérios maiores: piora da função renal; lesão hepatocelular aguda; rash cutâneo com necrose epidérmica tóxica (síndrome de Lyell), eritema multiforme ou dermatite esfoliativa difusa ou máculo-papular;

2. critérios menores: febre, eosinofilia e leucocitose. 
O diagnóstico de SHA requer história de exposição ao alopurinol, ausência de exposição a outros fármacos que possam causar quadro clínico similar e presença de dois critérios maiores ou um critério maior associado a um critério menor ${ }^{(8)}$.

A presença de rash morbiliforme é a apresentação inicial mais freqüente de reações graves, incluindo a síndrome de Lyell ${ }^{(8)}$. Podem ocorrer insuficiências hepática ou renal, geralmente reversíveis. O óbito relaciona-se freqüentemente com quadros sépticos ${ }^{(6,8)}$.

Destaca-se que o risco de desenvolvimento de SHA relaciona-se com o nível sérico de oxipurinol, metabólico terapêutico do alopurinol. Como o oxipurinol é excretado pelos rins, há maior taxa de SHA nos casos com redução da função renal ou em uso de tiazídicos, mas o exato mecanismo da síndrome ainda não é evidente ${ }^{(3,6)}$. Presume-se que o acúmulo de oxipurinol leve ao dano tecidual promovendo resposta imunológica com formação de anticorpos contra o tecido, caracterizando uma vasculite difusa em conseqüência de uma reação de hipersensibilidade do tipo III ${ }^{(3,4,6)}$.

Não há tratamento estabelecido, a não ser a suspensão da droga e instituição de terapêutica de suporte ${ }^{(6,8)}$. A hemodiálise foi sugerida como forma terapêutica, mas seu uso é restrito, visto que há casos de SHA com níveis indetectáveis de oxipurinol, o que mostra que esta substância não precisa estar presente para a persistência da síndrome ${ }^{(6)}$. Alguns autores também relatam bons resultados com técnicas de dessensibilização ao fármaco, mas parece-nos que o uso racional do alopurinol é a melhor terapêutica a ser adotada ${ }^{(6,9,10)}$.

\section{REFERÊNCIAS}

1. Roubenoff R: Gout and Hyperuricemia. Rheum Dis Clin Nor Am 16: 539-50, 1990.

2. Golding D: Managing Gout in Practice. The Practioner 240: 486-90, 1996.

3. Kumar A, Edward N, White MI, Johnston PW, Catto GRD: Allopurinol, Erythema Multiforme and Renal Insufficiency. BMJ 312: 173-5, 1996.

4. Gonzáles RP, Hernández RC: Síndrome de Hipersensibilidad al Alopurinol. Rev Clin Esp 201: 493, 2001.

5. Scott JT: Gout: the last 50 years. J R Soc Med 89: 634-7, 1996.

6. Pluim HJ, Van Deuren M, Wetzels JFM: The Allopurinol Hypersensivity Syndrome. Neth J Med 52: 107-10, 1998.

7. Ferraz MB, Sato EI, Nishie IA, Visioni RA: A Survey of Current Practices in Gouty Arthritis and Asymptomatic Hyperuricemia in São Paulo, Brazil. J Rheumatol 21: 374-5, 1994.

8. Pereira S, Almeida J, Silva AO, Quintas M, Candeias O, Freitas F: Necrose Hepática Fatal por Alopurinol. Acta Med Port 11: 1141-4, 1998.
A droga é indicada apenas nos seguintes casos:

- tratamento da gota primária com tofo ou litíase de ácido úrico causada por hiperprodução;

- litíase de ácido úrico ou de oxalato de cálcio sem gota, associada ao aumento na excreção urinária de urato;

- gota renal secundária com tofo;

- doença mieloproliferativa ou outra malignidade (preferencialmente antes do início de quimioterapia ou radioterapia);

- grande freqüência de surtos apesar do uso profilático com colchicina;

- intolerância a agentes uricosúricos;

- síndrome de Lesch-Nyhan ou doença de Von Gierke ${ }^{(3,6)}$.

Além da correta indicação terapêutica, devemos ter consciência da necessidade de ajuste da dose na redução da função renal, bem como o cuidado de saber quais medicamentos são utilizados concomitantemente pelo paciente ${ }^{(8,11)}$.

Outros tratamentos também não são isentos de riscos, com o exemplo da benzobromarona. Este fármaco deriva do benzofurano e atua reduzindo a absorção do ácido úrico nos túbulos renais, porém, já foram-lhe atribuídos óbitos em várias regiões do mundo, principalmente decorrentes de injúria hepática, bem como pela potencialização do efeito anticoagulante da warfarina ${ }^{(12,13)}$.

Desta forma, através do acompanhamento clínico freqüente do paciente e da atualização rotineira do médico quanto aos riscos e benefícios de cada droga hipouricêmica, poderemos reduzir o número de óbitos iatrogênicos.

9. Ortega JD, Trindade C, Llamazares AA, Bravo AC, Alvarez-Lovel MC, Martinez-Cócera C: Hypersensitivity to Allopurinol. Efficacy of a Desensitizing Protocol in 3 Cases. An Med Interna 18: 27-8, 2001.

10. Fam AG, Dunne SM, Iazzetta J, Paton TW: Efficacy and Safety of Desensitization to Allopurinol Following Cutaneous Reactions. Arthritis Rheum 44: 231-8, 2001.

11. Vásquez-Mellado J, Morales EM, Pacheco-Tena C, Burgos-Vargas R: Relation Between Adverse Events Associated with Allopurinol and Renal Function in Patients with Gout. Ann Rheum Dis 60: 981-3, 2001.

12. Wagayama $\mathrm{H}$, Shiraki $\mathrm{K}$, Sugimoto $\mathrm{K}$, et al: Fatal Fulminant Hepatic Failure Associated with Benzbromarone. J Hepatol 32: $874,2000$.

13. Takahashi H, Sato T, Shimoyama Y, et al: Potentiation of Anticoagulant Effect of Warfarin Caused by Enatioselective Metabolic Inhibition by the Uricosuric Agent Benzbromarone. Clin Pharmacol Ther 66: 569-81, 1999. 